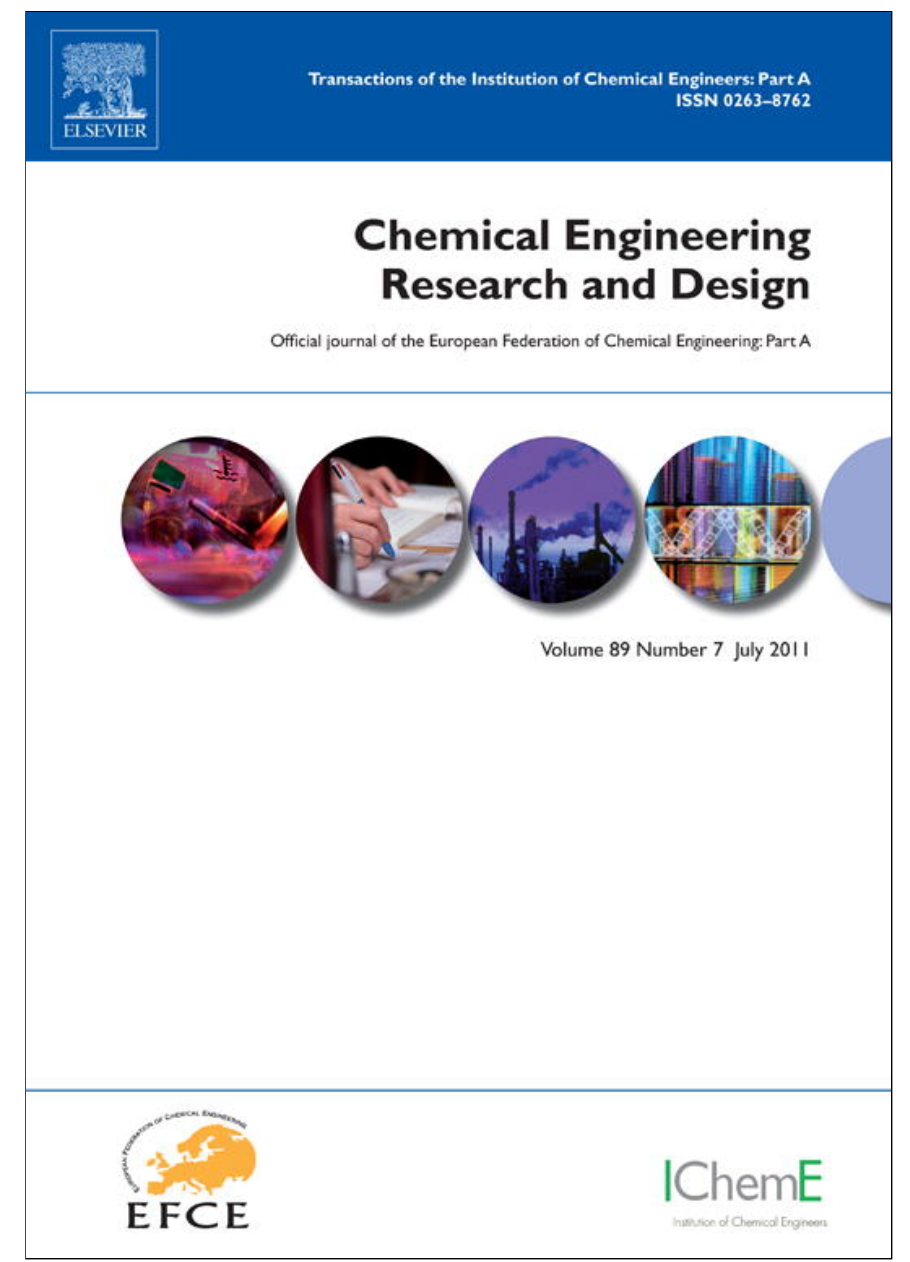

This article appeared in a journal published by Elsevier. The attached copy is furnished to the author for internal non-commercial research and education use, including for instruction at the authors institution and sharing with colleagues.

Other uses, including reproduction and distribution, or selling or licensing copies, or posting to personal, institutional or third party websites are prohibited.

In most cases authors are permitted to post their version of the article (e.g. in Word or Tex form) to their personal website or institutional repository. Authors requiring further information regarding Elsevier's archiving and manuscript policies are encouraged to visit:

http://www.elsevier.com/copyright 


\title{
Solution viscosity and flocculation characteristics of linear polymeric flocculants in various media
}

\author{
T.O. Chimamkpam ${ }^{a}$, M.G. Rasteiro ${ }^{a, *}$, F.A.P. Garcia ${ }^{a}$, E. Antunes ${ }^{a}$, P. Ferreira ${ }^{a}$, \\ D. Hunkeler ${ }^{b}$, C. Wandrey ${ }^{c}$ \\ a Chemical Engineering Department, Faculty of Science and Technology, University of Coimbra, 3030-790 Coimbra, Portugal \\ ${ }^{b}$ AQUA + TECH, Chemin du Chalet-du-Bac 4, CH-1283 La Plaine CP 28, Geneva, Switzerland \\ c Institut de Ingénierie Biologique et Institutes des Sciences et Ingénierie Chimiques, École Polytechnique Fédérale de Lausanne (EPFL), \\ CH-1015 Lausanne, Switzerland
}

\begin{abstract}
A B S T R A C T
Rheological characteristics of linear copolymers of acrylamide (AM) and acryloyloxyethyltrimethyl ammonium chloride (Q9), differing in molar mass and chemical composition, have been studied in distilled water (DW) and industrial water (IW) obtained from a paper mill. For all copolymers, the shear viscosity, $\mu$, was much lower as the ionic strength of the water increased, with near Newtonian behaviour observed at high ionic strengths. In DW, the polymer solutions were yield pseudoplastic. Comparison of the behaviour of all flocculants at the same shear rate in the two media was accomplished by modelling the rheological data.

The characteristics of the copolymers could be related with their flocculation performance in IW. The rate of flocculation in the IW was generally higher than in the DW. In general, flocculants with higher charge density were effective at lower concentrations exhibiting a lower value for the optimum PEL dosage. The flocculation data were supported with zeta potential measurements. In all cases flocs break under shear with an inability to recover the floc size with time. Overall, the polyelectrolyte behaviour in flocculation as a function of molar mass, charge density and quality of the medium could be correlated. This is of particular importance for papermaking due to the modern tendencies for water closure.
\end{abstract}

๑ 2010 The Institution of Chemical Engineers. Published by Elsevier B.V. All rights reserved.

Keywords: Flocculation; Cationic polyelectrolyte; Copolymers; Rheology; Papermaking

\section{Introduction}

Flocculants are preferably charged polymers used to facilitate separation processes. Cationic and anionic polymers, generally with molar masses above $10^{6} \mathrm{~g} / \mathrm{mol}$, cause colloidal matter to aggregate, forming particles large enough to sediment under gravity or submitted to other driving forces. The overall environmental impact of flocculation is important for global sustainable development since the downstream burdens associated with the water life cycle are also influenced. Cationic polymers usually involve quaternary ammonium compounds and protonated amines (Bourdillon et al., 2006; Kemer, 1988; Chen, 1993; Mortimer, 1991; Ayol et al., 2005).

In papermaking, the addition of flocculants is vital for controlling the equilibrium between the retention of fillers and the drainage of water on the wet-end of the paper machine. Usually, these flocculants are cationic polyelectrolytes, which can also be combined with non-ionic polymers or inorganic micro particles. The entire process of papermaking is typically performed under extremely harsh conditions. Thus, the initial properties of the flocculants could be subjected to modification. Such severe conditions include turbulence, which could cause high shearing, temperature, $\mathrm{pH}$ and ionic content of the circulating water (Gregory, 1985; Berlin and Kislenko, 1995; Berlin et al., 1997; Biggs et al., 2000). Hence, there is an important need for evaluating the behaviour and stability of polyelectrolytes under real process conditions. Such an evaluation was the aim of the study reported here, following studies from other authors which tried to relate the rheological properties of PEL solutions with the cationic content of the aqueous media (Lauten and Nystrom, 1999; Rashidi et al., 2010).

\footnotetext{
* Corresponding author. Tel.: +351 239798700; fax: +351 239798703.

E-mail address: mgr@eq.uc.pt (M.G. Rasteiro).

Received 12 March 2010; Received in revised form 22 September 2010; Accepted 20 October 2010 0263-8762/\$ - see front matter @ 2010 The Institution of Chemical Engineers. Published by Elsevier B.V. All rights reserved. doi:10.1016/j.cherd.2010.10.022
} 


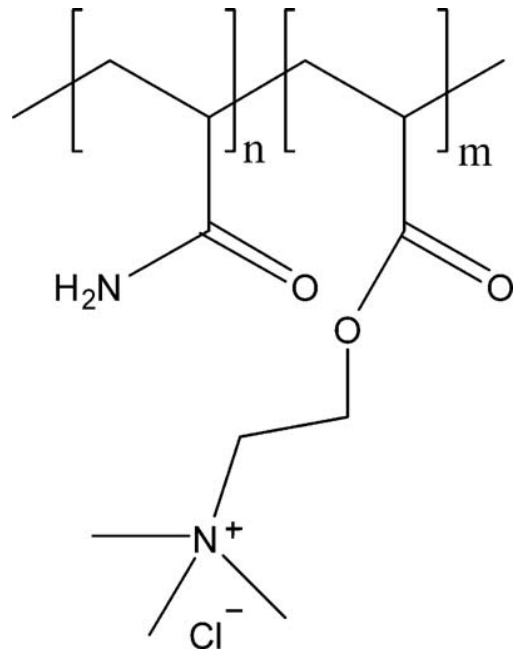

Fig. 1 - Chemical structure of the acrylamide/acryloyloxyethyltrimethylammonium chloride (AM/Q9) copolymers.

The behaviour of some commercially available flocculants was studied as a function of their chemical compositions and macromolecular characteristics not only in distilled water (DW), but also in a real industrial water (IW) from a paper mill, since it is well known that the polyelectrolyte conformation depends on the ionic content of the media (Jachimska et al., 2010). Subsequent to the rheological characterization, flocculation tests were performed in DW and IW. To support the correlation of the polymer solution flow behaviour with flocculation performance, the chemical compositions, macromolecular and electrochemical characteristics of the copolymers were analysed.

\section{Experimental}

\subsection{Materials}

The cationic flocculants: Alpine-Floc ${ }^{\mathrm{TM}} \mathrm{E} 1$, Alpine-Floc ${ }^{\mathrm{TM}} \mathrm{E} 2$, Alpine-Floc ${ }^{\mathrm{TM}} \mathrm{E} 4$, and Alpine-Floc ${ }^{\mathrm{TM}} \mathrm{G}$, all copolymers of acrylamide (AM), and acryloyloxyethyltrimethyl ammonium chloride (Q9) were supplied by AQUA + TECH (Switzerland) as emulsions. The E series are linear copolymers obtained by copolymerizing monomer batch weight compositions AM:Q9 in 1:1 ratio while G1 was obtained from AM:Q9 in 4:1 ratio. The chemical structure of the copolymers is schematically shown in Fig. 1.

The median size of the precipitated calcium carbonate (PCC) particles was $0.5 \mu \mathrm{m}$, measured with Laser Diffraction Spectroscopy (LDS) (Mastersizer 2000, Malvern Inst., UK).

\subsection{Copolymer isolation and solution preparation}

For the copolymer macromolecular characterization by dilution viscometry and composition analysis, the polyelectrolytes were isolated from the emulsions by repeated precipitation in acetone and re-dissolution in water. After complete evaporation of acetone, the samples were gently dried retaining about $10 \%$ residual humidity. The sample humidity was considered for the final precise solution concentration. The dry content was determined gravimetrically by vacuum drying a portion of each sample for $72 \mathrm{~h}$ at $45^{\circ} \mathrm{C}$ in the presence of $\mathrm{P}_{2} \mathrm{O}_{5}$.
Solutions for dilution viscometry were prepared with $0.05 \mathrm{M}$ and $0.1 \mathrm{M} \mathrm{NaCl}$ as solvents. To avoid basic hydrolysis of the ester group (Babazadeh, 2006; Ochoa et al., 2007), the samples were dissolved in acidic water (pH 3.5 adjusted with $\mathrm{HCl}$ ) and then mixed with double concentrated $\mathrm{NaCl}$ acidic solution (volume ratio1:1).

\subsection{Methods}

\subsubsection{Dilution viscometry}

The intrinsic viscosity, $[\eta]$, of samples dissolved in $0.05 \mathrm{M}$ and $0.1 \mathrm{M} \mathrm{NaCl}$ was determined at $20 \pm 0.1^{\circ} \mathrm{C}$ using a Viscologic TI1 capillary viscometer, capillary $0.58 \mathrm{~mm}$ (Sematech, Nice France). $[\eta]$ was obtained as the intercept from plots according to Schulz and Blaschke (1941) and Huggins (1942).

\subsubsection{Analysis of the copolymer composition}

The compositions of the copolymers were analysed by argentometric titration using a 736 GP Titrino (Metrohm, Switzerland). Potentiometric titration of $\mathrm{AM} / \mathrm{Q} 9$ copolymer solutions of known copolymer dry content directly yields the copolymer composition.

\subsubsection{Characterisation of the industrial water}

The composition of the IW collected from the paper mill was analysed by the Atomic Absorption technique (PerkinElmer 3300, USA).

The conductivity and $\mathrm{pH}$ were measured with a conductivity meter (Crison Inst., Spain, Basic 30) and pH meter (Hanna Inst., USA, 8417), respectively.

\subsubsection{Samples for flocculation tests}

Solutions containing $1 \mathrm{wt}$ \% of the flocculants were prepared from the original emulsions with DW and IW. Prior to use, the IW was filtered under vacuum using a $0.2 \mu \mathrm{m}$ pore size membrane, to remove traces of suspended material. For all media, the flocculants were added drop-wise, first to half of the total volume and shacken vigorously. Subsequently the remaining half of the water was added gradually, shaken till the solution became homogeneous, and left over night before use. Solutions were freshly prepared daily.

Suspensions of $1 \mathrm{wt} . \%$ of the PCC were prepared in DW and IW. The suspensions were first mixed by magnetic stirring and subsequently sonicated at $50 \mathrm{kHz}$ for $15 \mathrm{~min}$ to obtain a good dispersion of the PCC particles. The pH of the PCC suspensions, prepared under these conditions, was 7.5. The zeta potential was measured with a Zetasizer ZS (Malvern Inst., $\mathrm{UK}$ ) and the values were $-30 \mathrm{mV}$ and $-37 \mathrm{mV}$ in DW and IW, respectively.

\subsubsection{Rheological measurements}

Temperature controlled rheological characterizations of all flocculants were carried out using a Brookfield programmable viscometer DV-II+ with coaxial cylinders configuration. Spindle 18 was used for all measurements, which were repeated five times. Each experimental set involved measuring the solution viscosity at 25 and $40^{\circ} \mathrm{C}$ for a shear rate range from 5 to $264 \mathrm{~s}^{-1}$.

\subsubsection{Flocculation experiments with E1 and G1 in distilled} and industrial water

The flocculation, deflocculation and reflocculation using E1 and G1 as flocculant, both in DW and IW were carried out following the same procedure reported by Rasteiro et al. (2008a, 
Table 1 - Chemical composition and intrinsic viscosity of the flocculants according to Huggins and Schulz-Blaschke: $[\eta]_{H}$ and $[\eta]_{\mathrm{SB}}\left(\mathrm{k}_{\mathrm{H}}\right.$ : Huggins parameter, $\mathrm{k}_{\mathrm{SB}}$ : Schulz-Blaschke parameter).

\begin{tabular}{|c|c|c|c|c|c|c|c|c|c|}
\hline \multirow[t]{2}{*}{ Sample } & \multirow[t]{2}{*}{ Q9 (wt.\%) } & \multicolumn{4}{|c|}{$0.05 \mathrm{M} \mathrm{NaCl}$} & \multicolumn{4}{|c|}{$0.1 \mathrm{M} \mathrm{NaCl}$} \\
\hline & & {$[\eta]_{\mathrm{H}}(\mathrm{mL} / \mathrm{g})$} & $k_{\mathrm{H}}$ & {$[\eta]_{\mathrm{SB}}(\mathrm{mL} / \mathrm{g})$} & $k_{\mathrm{SB}}$ & {$[\eta]_{\mathrm{H}}(\mathrm{mL} / \mathrm{g})$} & $k_{\mathrm{H}}$ & {$[\eta]_{\mathrm{SB}}(\mathrm{mL} / \mathrm{g})$} & $k_{\mathrm{SB}}$ \\
\hline E1 & 45.5 & 2505 & 0.12 & 2515 & 0.11 & 1679 & 0.26 & 1692 & 0.21 \\
\hline E2 & 46.2 & 1558 & 0.20 & 1564 & 0.17 & 1304 & 0.30 & 1312 & 0.25 \\
\hline E4 & 46.5 & 1250 & 0.08 & 1250 & 0.08 & 808 & 0.28 & 810 & 0.25 \\
\hline G1 & 19.0 & 1510 & 0.51 & 1547 & 0.35 & 1154 & 0.81 & 1188 & 0.53 \\
\hline
\end{tabular}

2008b). LDS was used to monitor the flocculation process. The PCC suspension was used for the flocculation tests in DW and IW with a concentration of $0.05 \%$ (w/w). Flocculation was conducted under turbulence, the stirring conditions having been kept constant throughout the tests, with an average shear rate in the vessel of $312 \mathrm{~s}^{-1}$. The volume of the suspension in the LDS equipment vessel was $700 \mathrm{ml}$. The flocculation kinetic curve, optimum flocculant concentration, floc structure and floc resistance were determined with the LDS equipment (Rasteiro et al., 2008a, 2008b).

\section{Results and discussion}

\subsection{Intrinsic viscosity and copolymer composition analyses}

Table 1 summarizes the chemical composition of the copolymers and the results of the dilution viscometry.

The analysed cationic monomer content of the E series copolymers is approximately $46 \mathrm{wt}$.\%. It is slightly lower than what the supplier indicated (50wt.\%). The reason could be incomplete quaternization of the cationic monomer used to produce the copolymers. The same holds for the G1 sample for which 20 wt.\% cationic monomer content was indicated.

As intended for the E series, the intrinsic viscosity, $[\eta]$, which correlates with the molar mass according to the relation: $[\eta]=K_{\eta} M_{\eta}^{a}$, is in the order E1 $>E 2>E 4$ for the two ionic strengths $(0.05$ and $0.1 \mathrm{M} \mathrm{NaCl})$. However, as expected for such polyelectrolytes, $[\eta]$ is lower at higher ionic strength. Nevertheless, the Schulz-Blaschke and Huggins coefficients, $k_{\mathrm{H}}$ and $k_{\mathrm{SB}}$, confirm good solubility in $0.1 \mathrm{M} \mathrm{NaCl}$. Comparing these coefficients for $\mathrm{G} 1$ in 0.05 and $0.1 \mathrm{M} \mathrm{NaCl}$, the decrease of the solvent goodness with increasing ionic strength becomes visible.

It should be noted that the ionic strengths of the dilution viscometry $(0.05$ and $0.1 \mathrm{~mol} / \mathrm{L})$ are much higher than the ionic strength of the IW, as will be presented in a subsequent section.

\subsection{Chemical analysis and characteristics of the media}

Table 2 presents the results of the chemical analysis, the conductivity and the $\mathrm{pH}$ of the DW and IW. The much higher conductivity of the IW results from the ionic content of the circulating water in the papermaking process.

\subsection{Rheological behaviour of the flocculants in} distilled water at $25^{\circ} \mathrm{C}$

Figs. 2 and 3 show the rheograms (shear stress versus shear rate and apparent viscosity versus shear rate) of all flocculants in DW at $25^{\circ} \mathrm{C}$. All flocculants displayed a yield pseudoplastic behaviour, exhibiting a lower apparent viscosity at higher shear rates. The apparent viscosity of a shear thinning fluid decreases with rising shear rate from the zero shear viscosity to the infinite shear viscosity. With increasing shear rate, the macromolecules are progressively aligned, instead of the random intermingled state which exists when the fluid is at rest. The major molecule axes are brought into line with the direction of flow and the viscosity decreases (Chhabra and Richardson, 1999).

E1 and G1 exhibited higher viscosity and more clearly yield pseudo-plastic behaviour than E2 and E4. Despite the lower [ $\eta]$ of G1 compared to E2, the solution viscosity of the G1 copolymer is higher. This likely results from the lower charge density along the G1 copolymer chains (see Table 1), which leads to less extended coils in 0.05 and $0.1 \mathrm{~mol} / \mathrm{L} \mathrm{NaCl}$ solution, while in DW the chain is more extended reflecting better the molar mass when compared to the E PEL series, since less interaction between the chain charges is present.

For the E series, the viscosity decrease of E1 as shear rate increases was most pronounced decreasing from 311 to 58 $\mathrm{mPa}$ s, followed by E2, from 89 to $29 \mathrm{mPa}$ s, and then E4 from 40 to $19 \mathrm{mPa}$ s. Thus, for $\mathrm{E} 2$ and in particular for $\mathrm{E} 4$, the behaviour was close to Newtonian.

\subsection{Rheological behaviour of the flocculants in industrial water at $25^{\circ} \mathrm{C}$}

All flocculants demonstrated much lower viscosity in the IW (Fig. 4) in comparison to the DW (Fig. 3). In general, the rheology of all polymer solutions was still yield pseudo-plastic, though in the case of E2, E4 and G1, the behaviour was closer to Newtonian. The presence of not quantified colloidal components, in addition to the inorganic salts in the IW (Rasteiro et al., 2008b) may further influence the copolymer conformation and interaction and consequently, the viscosity (see Lauten and Nystrom, 1999). The influence was more important for E1, the polymer with higher $[\eta]$. E2 and E4 behaved similarly in both media. So, lower molar mass PELs proved to be less affected by the medium composition.

Table 2 - Ionic content, conductivity and pH of industrial water (IW) and distilled water (DW).

\begin{tabular}{llllllllll} 
Water & $\mathrm{Ca}^{2+}$ & $\mathrm{Mg}^{2+}$ & $\mathrm{K}^{+}$ & $\mathrm{Na}^{+}(\mathrm{mg} / \mathrm{L})$ & $\mathrm{Al}^{3+}$ & $\mathrm{Cl}^{-}$ & $\mathrm{SO}_{4}^{2-}$ & $\kappa(\mu \mathrm{S} / \mathrm{cm})$ & $\mathrm{pH}$ \\
\hline IW & 46 & 6 & 4 & 101 & 0.4 & 153 & 32 & 734 & 7.8 \\
DW & & & & & & & & 15.1 & 5.9 \\
\hline
\end{tabular}




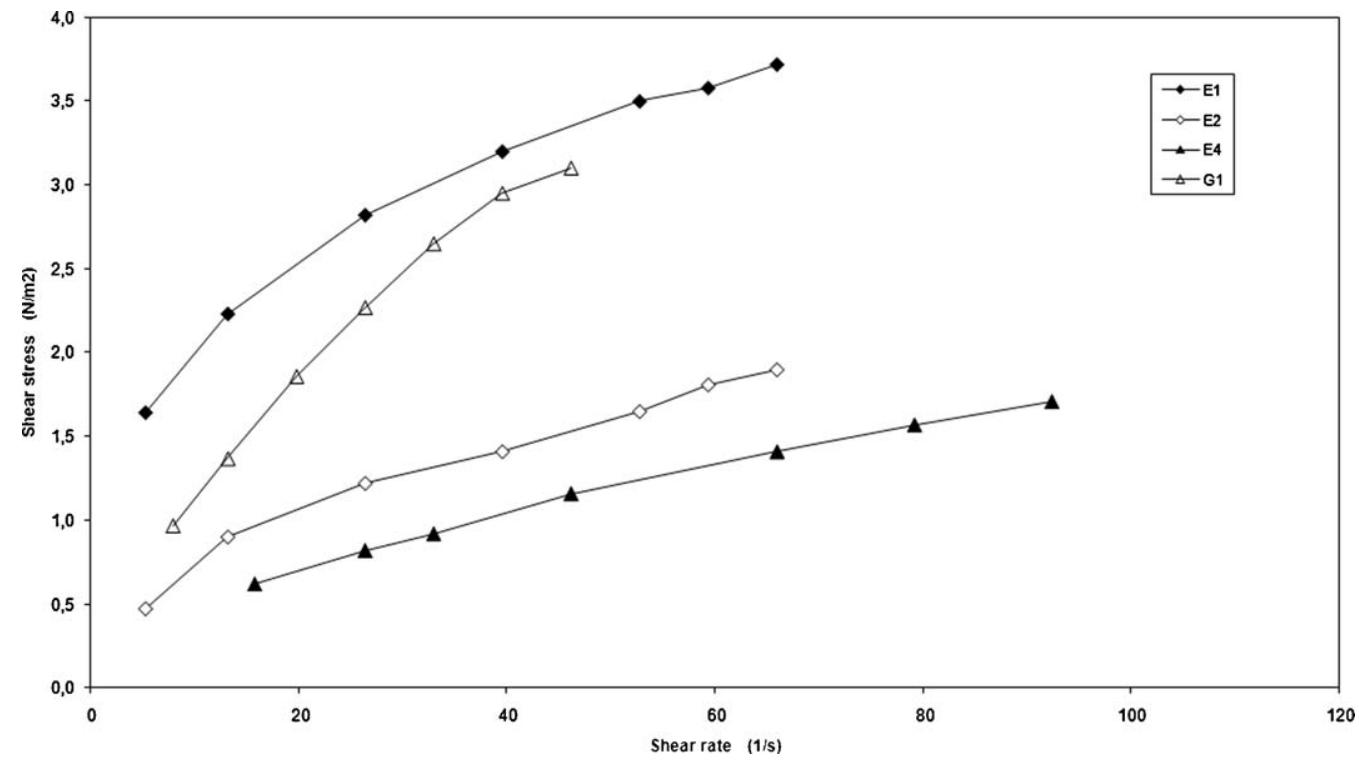

Fig. 2 - Shear stress versus shear rate: E1, E2, E4 and G1 in the distilled water at $25^{\circ} \mathrm{C}$.

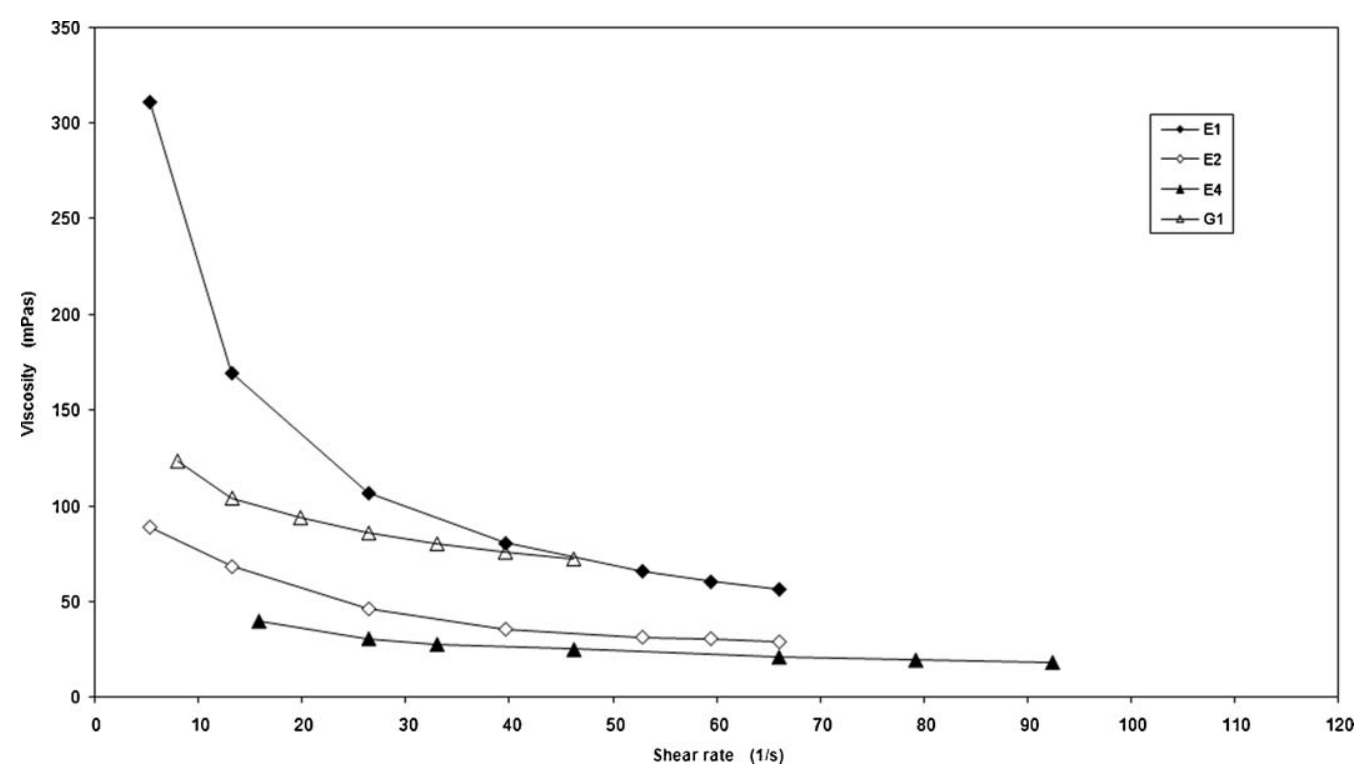

Fig. 3 - Viscosity versus shear rate: E1, E2, E4 and G1 in the distilled water at $25^{\circ} \mathrm{C}$.

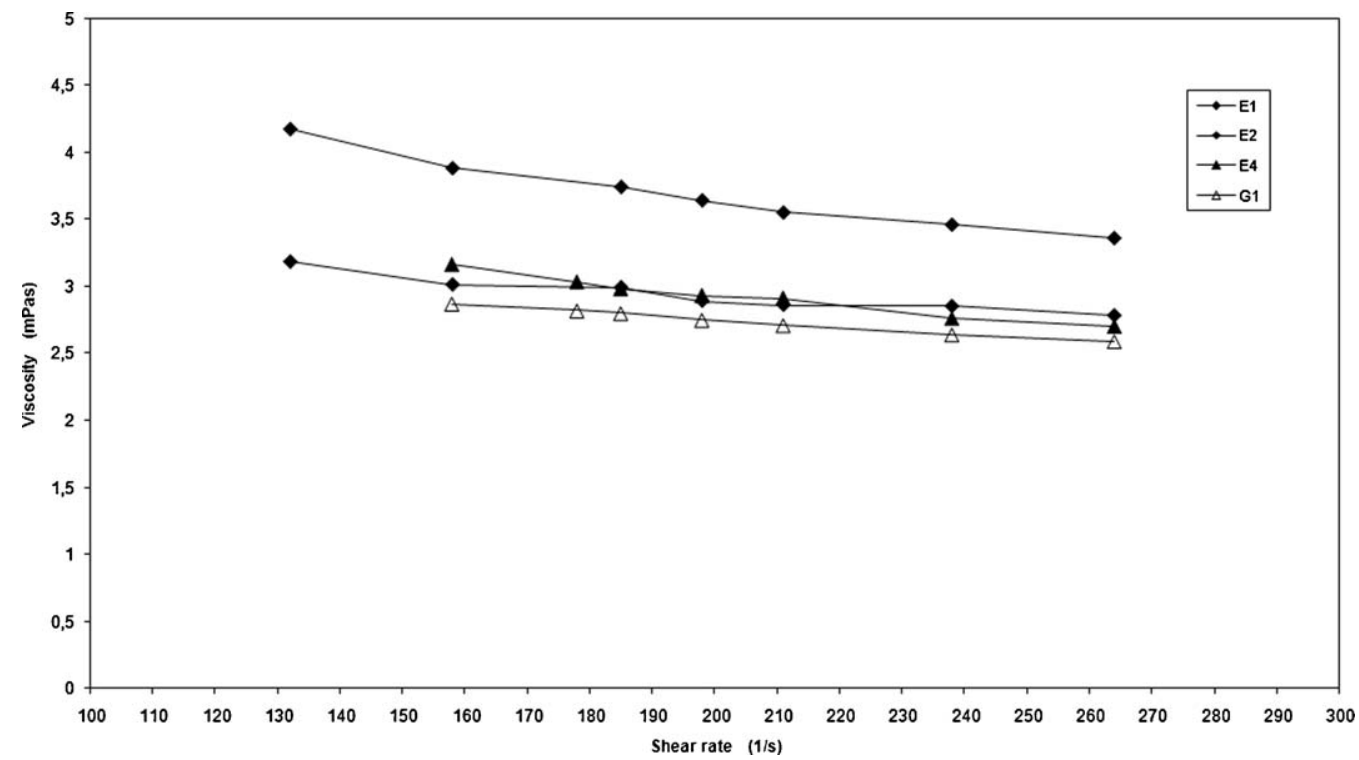

Fig. 4 - Viscosity versus shear rate: E1, E2, E4 and G1 in the industrial water at $25^{\circ} \mathrm{C}$. 


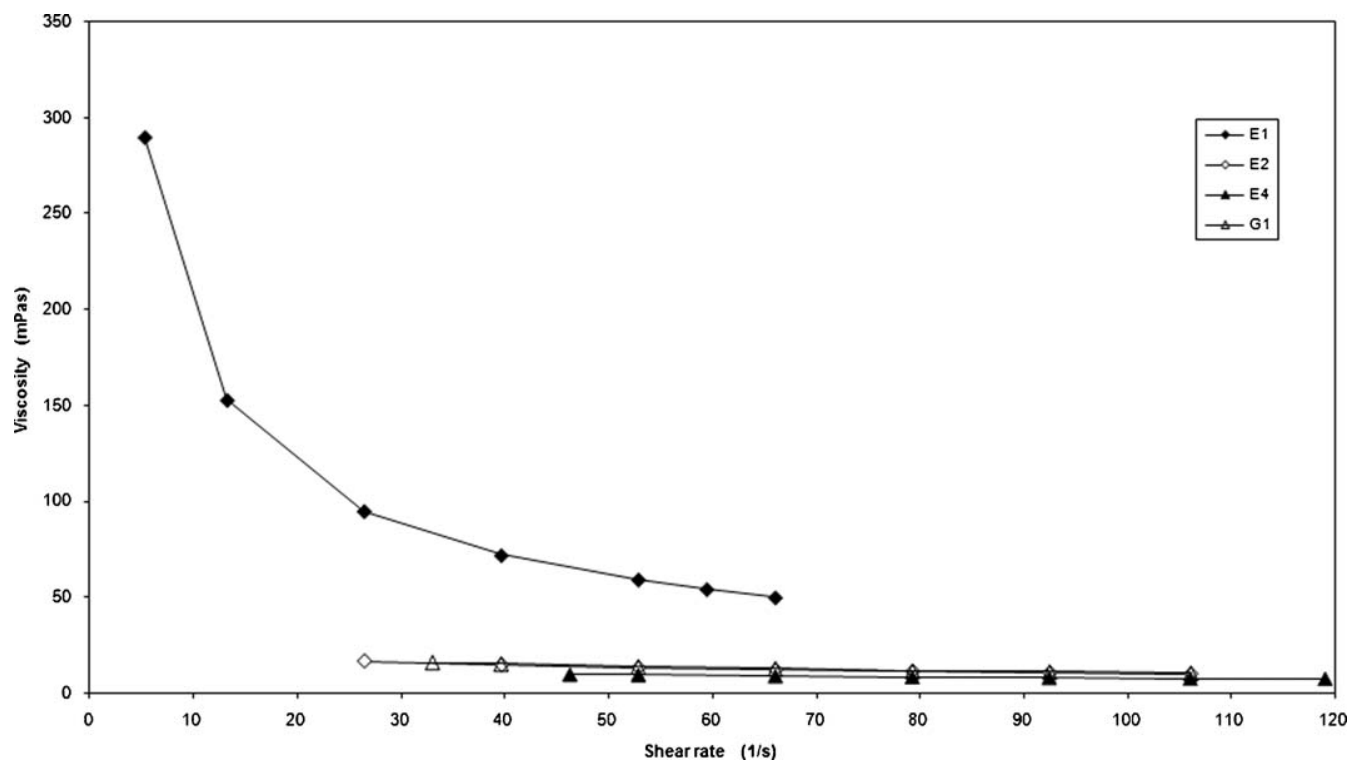

Fig. 5 - Viscosity versus shear rate: E1, E2, E4 and G1 in the distilled water at $40{ }^{\circ} \mathrm{C}$.

\subsection{Temperature dependence of the viscosity}

The viscosity of most fluids decreases with increasing temperature. Accordingly, the viscosity of the flocculants studied decreased for the highest temperature (compare Figs. 3 and 5 and Figs. 4 and 6). All flocculants were tested in DW and IW at 25 and $40^{\circ} \mathrm{C}$. In all cases, a simultaneous decrease in yield stress and viscosity at infinite shear rate was observed at the higher temperature. The differences were more pronounced in the DW. At $40^{\circ} \mathrm{C}$, the apparent viscosity of E1 in DW varied from 290 to $50 \mathrm{mPas}$, as shear rate increased, lower than the values at $25^{\circ} \mathrm{C}, 311-57 \mathrm{mPa}$, for the same shear rate span. For E2 in DW, the effect of temperature was more prominent. For a shear rate interval between 25 and $65 \mathrm{~s}^{-1}$, the apparent viscosity varied from 46 to $29 \mathrm{mPa}$ s at $25^{\circ} \mathrm{C}$ and $17-12 \mathrm{mPa}$ s at $40^{\circ} \mathrm{C}$. E4 likewise displayed a significant decrease of the viscosity at higher temperature. For a shear rate interval between 45 and $95 \mathrm{~s}^{-1}$, the apparent viscosity varied from 25 to $19 \mathrm{mPa}$ s at $25^{\circ} \mathrm{C}$ and $10-8 \mathrm{mPa}$ s at $40^{\circ} \mathrm{C}$. The effect of temperature on the rheological behaviour of G1 was also significant. For a shear rate interval between 30 and $50 \mathrm{~s}^{-1}$, the apparent viscosity varied from 80 to $72 \mathrm{mPas}$ at $25^{\circ} \mathrm{C}$ and $16-14 \mathrm{mPas}$ at $40^{\circ} \mathrm{C}$ as shear rate increased.

Due to equipment limitations, the shear rate interval could not be kept constant for all measurements because a certain level of signal to noise ratio must always exist for an accurate measurement (torque percentage should always be above $10 \%)$.

The major observable feature in Figs. 3 and 5 is that increasing the temperature from 25 to $40^{\circ} \mathrm{C}$ led to an almost Newtonian behaviour of the solutions of the flocculants E2, E4 and G1 in the DW. The influence of temperature was much less evident for E1, which is the copolymer with the highest $[\eta]$.

For the solutions in IW, the decrease of the viscosity, with increasing shear rate, of all flocculants at $40^{\circ} \mathrm{C}$, was slightly less than at $25^{\circ} \mathrm{C}$ (Figs. 4 and 6). As in DW, the behaviour of E2, E4 and G1 became closely Newtonian at higher temperature. Moreover, the influence of temperature was less pronounced in IW than in DW. This was because the initial viscosity at $25^{\circ} \mathrm{C}$ was already much lower than in DW and the rheological behaviour of E2, E4 and G1 almost Newtonian.

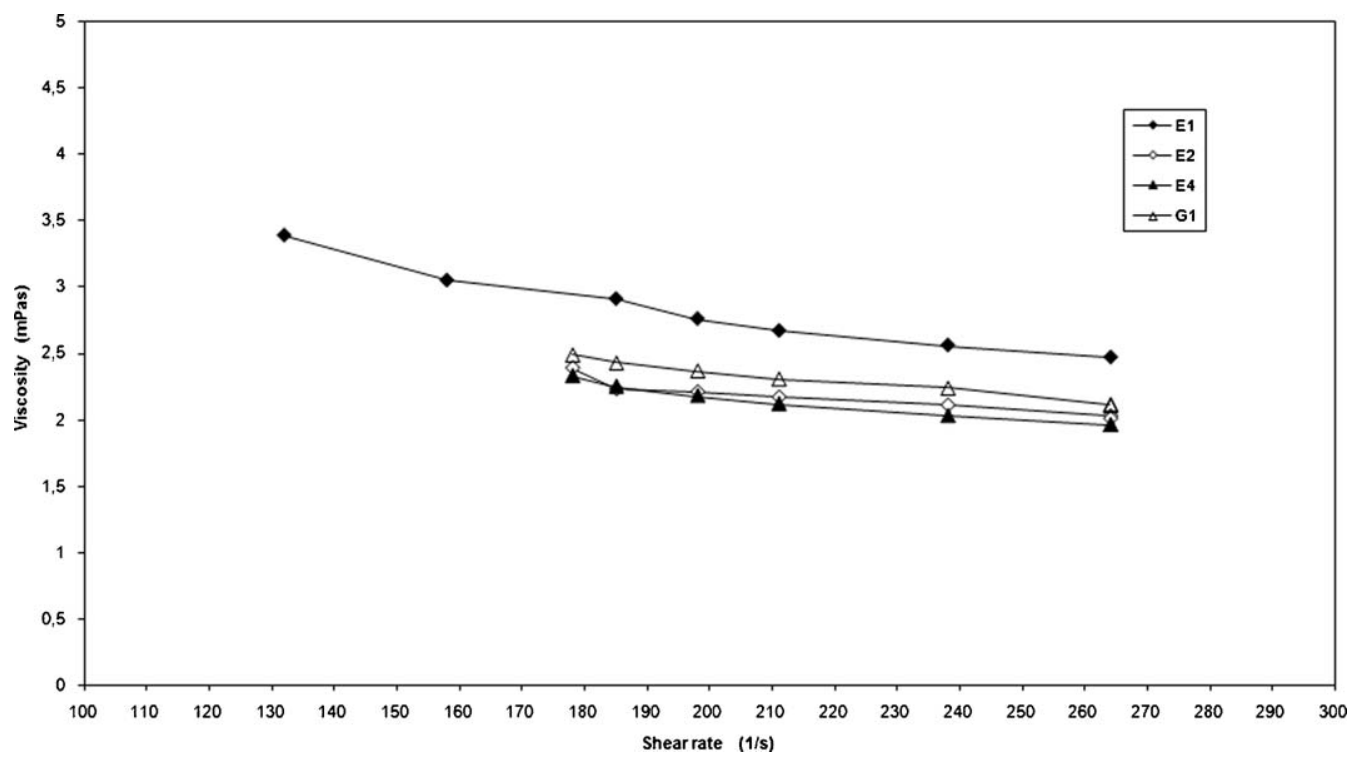

Fig. 6 - Viscosity versus shear rate: E1, E2, E4 and G1 in the industrial water at $40^{\circ} \mathrm{C}$. 


\begin{tabular}{|c|c|c|c|c|c|}
\hline \multirow[t]{2}{*}{ Sample } & \multicolumn{3}{|c|}{ DW } & \multicolumn{2}{|r|}{ IW } \\
\hline & $\gamma\left(\mathrm{s}^{-1}\right)$ & $\tau\left(\mathrm{N} / \mathrm{m}^{2}\right)$ & $\mu$ (mPa s) & $\tau\left(\mathrm{N} / \mathrm{m}^{2}\right)$ & $\mu(\mathrm{mPa} s)$ \\
\hline \multirow[t]{2}{*}{ E1 } & 10 & 2.05 & 0.205 & 0.16 & 0.016 \\
\hline & 100 & 4.37 & 0.044 & 0.47 & 0.0047 \\
\hline \multirow[t]{2}{*}{ E2 } & 10 & 0.61 & 0.061 & 0.10 & 0.01 \\
\hline & 100 & 2.21 & 0.022 & 0.33 & 0.0033 \\
\hline \multirow[t]{2}{*}{ E4 } & 10 & 0.48 & 0.048 & 0.11 & 0.011 \\
\hline & 100 & 1.75 & 0.018 & 0.37 & 0.0037 \\
\hline \multirow[t]{2}{*}{ G1 } & 10 & 1.15 & 0.115 & 0.11 & 0.011 \\
\hline & 100 & 6.06 & 0.061 & 0.33 & 0.0033 \\
\hline
\end{tabular}

3.6. Mathematical model for the yield pseudoplastic fluid behaviour

To compare the shear stress $(\tau)$ at the same shear rate $(\dot{\gamma})$ for all flocculants in the different media, the rheograms were adjusted to an equation using the Herschel-Bulkley model (Herschel and Bulkley, 1926)

$\tau=\tau_{y}+k \dot{\gamma}^{n}$

The yield stress $\left(\tau_{y}\right)$ was first extrapolated from the rheogram by fitting a polynomial of 2 nd order. Afterwards, the rheogram was fitted to Eq. (1) using the Curve Expert 1.3 software. Knowing the values of $\tau_{y}, k$ (consistency index) and $n$ (flow behaviour index), the shear stress at $\dot{\gamma} 10$ and $100 \mathrm{~s}^{-1}$ were calculated for each flocculant in the three media. Likewise the apparent viscosity $(\mu)$ was calculated for these shear rates (Table 3).

From Table 3, the values of $\tau$ and $\mu$ at a shear rate of $10 \mathrm{~s}^{-1}$ in the DW decreased in the order: E1 $>\mathrm{G} 1>\mathrm{E} 2>\mathrm{E} 4$ according to the previous observations, whereas in the IW, the $\tau$ and $\mu$ of E2, G1 and E4 were almost the same with only that of E1 being significantly higher.

At $100 \mathrm{~s}^{-1}$ in the DW, all the flocculants exhibited very similar values for both $\tau$ and $\mu$. This was already apparent from the rheograms since the major difference in the rheological behaviour, mainly between E1 and G1, is in the yield stress and not in the viscosity at infinite shear rate.

For the IW, the $\tau$ and $\mu$ of E1 were higher, followed by that of E2, E4 and G1, which show similar values of $\tau$ and $\mu$ at $100 \mathrm{~s}^{-1}$ as at $10 \mathrm{~s}^{-1}$

\subsection{Flocculation of PCC with E1 and G1 in distilled and industrial water: relationship with viscosity}

Here, previously reported data on the flocculation of E1 in both the DW and IW (Rasteiro et al., 2008b) will be used for comparison with G1. In both media, the correlation between the viscosity of the copolymer solutions of E1 and G1 and flocculation performance is the subject of discussion.

Fig. 7 shows the flocculation kinetic curves of PCC with E1 in both DW and IW. The optimum polymer concentration, which is defined as the one leading to the largest flocs, was much higher in IW than in DW (20 and $4 \mathrm{mg}$ of copolymer/g of PCC respectively).

For the lower charged G1 (Fig. 8), the same tendency was observed, with the optimum polymer concentration being $50 \mathrm{mg} / \mathrm{g}$ of PCC in IW and only $10 \mathrm{mg} / \mathrm{g}$ of PCC in DW. There-

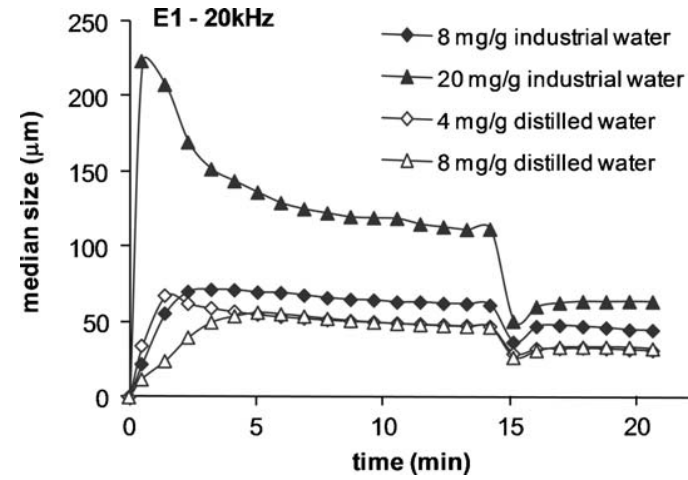

Fig. 7 - Flocculation of PCC with E1, deflocculation and reflocculation after sonication (at $15 \mathrm{~min}$ ) in the distilled and industrial water (Rasteiro et al., 2008b).

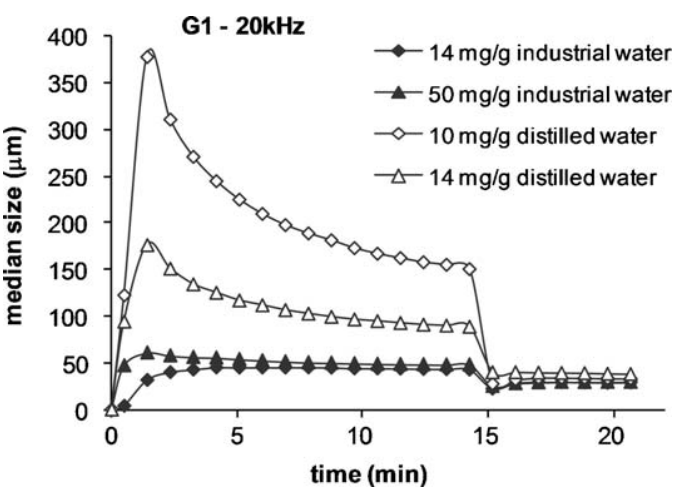

Fig. 8 - Flocculation of PCC with G1, deflocculation and reflocculation after sonication (at $15 \mathrm{~min}$ ) in the distilled and industrial water.

fore, in this particular system, the optimum concentration increases as the charge on the polymer is reduced.

This is reasonable since the IW contains several contaminants, the surface charge of the precipitated calcium carbonate particles in the IW will be more negative than in the DW and so more copolymer is required to neutralize those charges (Vanerek et al., 2000). The difference of the zeta potential of the PCC in DW and IW can be observed in Figs. 9 and 10.

In IW the polymer becomes more coiled (Jachimska et al., 2010). Subsequently, in the IW, a larger amount of polyelectrolyte was required to obtain the same particle surface coverage with the copolymer. Higher concentration was required for $\mathrm{G} 1$ than for $\mathrm{E} 1$ because of the lower content of cationic monomer units in G1. Nevertheless, it is interesting

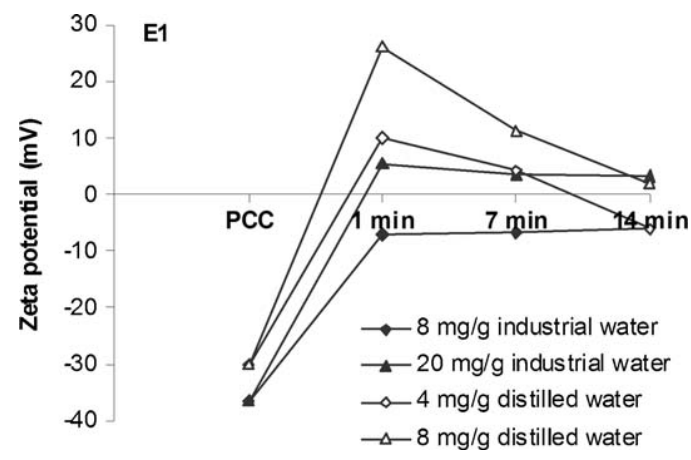

Fig. 9 - Zeta Potential of precipitated calcium carbonate suspension during flocculation with $\mathrm{E} 1$ in DW and IW: three different times after addition of flocculant (Rasteiro et al., 2008b). 


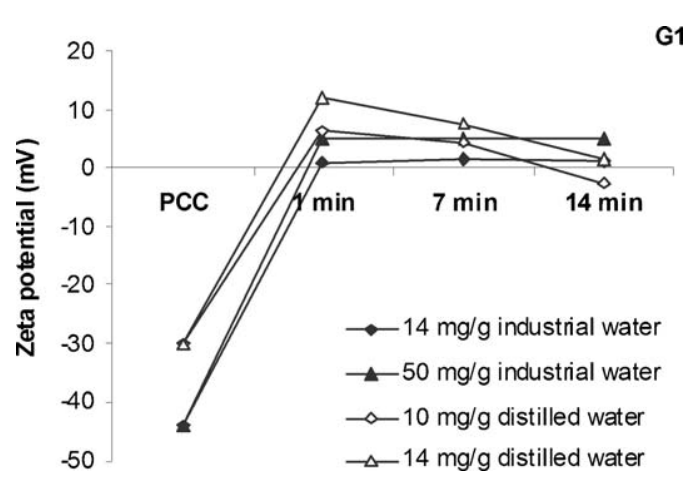

Fig. 10 - Zeta Potential of precipitated calcium carbonate suspension during flocculation with G1 in DW and IW: three different times after addition of flocculant.

to notice that the optimum dosage increased by a factor of five for both copolymers.

Moreover, polymer adsorption is enhanced by reducing the salt concentration (Stoll and Chodanowski, 2002; Shubin and Linse, 1997; Bremmell et al., 1998). Thus, for both flocculants, flocculation was faster in IW than in DW and this was more apparent for E1. This must be also related to the fact that repulsion between the particles decreased in the IW due to the depletion of the electric double layer resulting from the higher cationic content. This also agrees with the different viscosities of the flocculant solutions in both media since E1 and G1 had lower viscosities in the IW, especially E1.

The lower viscosity of the copolymer solutions in the IW is related to the reduced Debye length and to the resulting lower hydrodynamic radius of the copolymers in the medium. Hydrolysis of the copolymer molecules at the relatively high $\mathrm{pH}$ could also have an impact. Due to the lower hydrodynamic radius (Jachimska et al., 2010), the degree of coverage is reduced hence requiring higher polymer concentration for flocculation, as referred previously. Though higher polymer concentration was used for flocculation, the zeta potential was lower in IW (see Figs. 9 and 10) as already shown by Rasteiro et al. (2008b).

Regarding the size of the flocs there is an increase of the size in IW with E1, while the size decreases, comparing the results in DW and IW, with G1. The increase of the cationic content of the media can have a double effect: on the one hand reduction of the electric double-layer thickness favours aggregation and larger flocs are expected, on the other hand flocs can be more compact and become smaller. Thus, there is not a monotonic trend regarding the variation of the flocs size with the ionic content of the medium, in line with the results presented in Figs. 7 and 8. This tendency can be observed either if we compare results for the optimum PEL concentration for each medium or for a common concentration value (Figs. 7 and 8). Moreover, when the charge density is low the polymer conformation becomes less extended in IW. This corresponds to a lower hydrodynamic radius and, thus, smaller flocs are obtained.

Additionally, because of the relatively high $\mathrm{pH}$ of the IW, hydrolysis of the copolymer chains can occur. This effect is expected to be more significant in the case of G1, having an intrinsic lower number of charges per chain, resulting in a lower activity of this polymer in IW and leading, then, to smaller aggregates.

Floc resistance was also studied based on the methodology discussed in Rasteiro et al. (2008b) applying ultrasound $(20 \mathrm{kHz})$ to the flocs at $15 \mathrm{~min}$ over a period of $30 \mathrm{~s}$. Sonication

\begin{tabular}{|c|c|c|c|c|}
\hline \multirow[t]{2}{*}{ Polymer } & \multicolumn{2}{|c|}{ Concentration (mg/g) } & \multicolumn{2}{|c|}{ Break up $\%$ at $20 \mathrm{kHz}$} \\
\hline & DW & IW & DW & IW \\
\hline \multirow[t]{2}{*}{ E1 } & $4^{a}$ & 8 & 39 & 40 \\
\hline & 8 & $20^{\mathrm{a}}$ & 43 & 55 \\
\hline \multirow[t]{2}{*}{ G1 } & $10^{\mathrm{a}}$ & 14 & 82 & 48 \\
\hline & 14 & $50^{\mathrm{a}}$ & 55 & 47 \\
\hline
\end{tabular}

leads to floc fragmentation. Figs. 7 and 8 show the breakage and reflocculation stages after sonication. The effect of sonication was similar in both media and flocs break up was mainly related to flocs size. Larger flocs are more susceptible to breakage (Table 4). In the case of E1, floc break up was higher in IW since these flocs are larger, while for G1 the opposite was the case because larger flocs were obtained in the DW.

Reflocculation was generally low for both flocculants, especially in the DW, possibly because bridging is the main flocculation mechanism. Hence, floc breakage resulted from the detachment of the copolymer chains from the particles leading to rupture of bonds between the particles in the aggregate. However, reflocculation was somewhat higher in the IW for both E1 and G1 though more prominent in E1. The lower viscosity of E1 in the IW, which can be related to the more coiled conformation of the polymer, and a decrease in the significance of the bridging mechanism, explains the more extensive reflocculation of E1 in the IW.

\section{Conclusions}

The influence of the medium quality on the solution viscosity and rheological behaviour was quantified for four flocculants varying either in regards to the molar mass or the charge density. The differences observed when using DW and IW likewise governed the optimum flocculant dosage and the performance during the PCC particles flocculation.

A good correlation between copolymer composition, macromolecular characteristics, solution behaviour, and performance in flocculation was identified. Furthermore, analyses of the performance of E1 and G1 in the flocculation of precipitated calcium carbonate in both the DW and IW showed that larger dosages of E1 and G1 were required in the IW. Focculation was faster in the IW due to the attenuation of the repulsive forces between the PCC particles. In IW the viscosity of the copolymer solutions decreased remarkably, approaching an almost Newtonian behaviour. The effect was more pronounced for the copolymer of higher intrinsic viscosity and cationic charge content (E1).

The strategy of testing the rheological behaviour of the flocculants in different media proved to be important in assessing their performance during flocculation. It was confirmed that for proper evaluation of flocculant performance testing only in DW is insufficient. Instead, the medium intended for the practical flocculation should preferably be used in the tests. This can be particularly important in the case of papermaking, which was taken here as example, since water closure strategies contribute to dramatic changes in the suspending medium characteristics which influence the flocculation processes. Nevertheless, characterization in DW can provide 
preliminary information which remains useful in flocculant evaluation.

\section{Acknowledgements}

The authors greatly acknowledge the financial support of the Fundação para a Ciência e Tecnologia, Portugal (Grant PTDC/EQU-EQU/66669/2006).

\section{References}

Ayol, A., Dentel, S.K., Filibeli, A., 2005. Dual polymer conditioning of water treatment residuals. J. Environ. Eng. 131, 11321138.

Babazadeh, M., 2006. Synthesis and study of controlled release of ibuprofen from the new acrylic type polymers. Int. J. Pharm. 316, 68-73.

Berlin, A.A., Kislenko, V.N., 1995. Kinetic-models of suspension flocculation by polymers. Colloid Surf. A: Physicochem. Eng. Aspects A 104, 67-72.

Berlin, A.A., Solomentseva, I.M., Kislenko, V.N., 1997. Suspension flocculation by polyelectrolytes: experimental verification of a developed mathematical model. J. Colloid Interf. Sci. 191, 273-276.

Biggs, S., Habgood, M., Jameson, G.J., Yan, Y.D., 2000. Aggregate structures formed via a bridging flocculation mechanism. Chem. Eng. J. 80, 13-22.

Bourdillon, L., Hunkeler, D., Wandrey, C., 2006. The analytical ultracentrifuge for the characterisation of polydisperse polyelectrolytes. Prog. Colloid Polym. Sci. 131, 141-149.

Bremmell, K.E., Jameson, G.J., Biggs, S., 1998. Polyelectrolyte adsorption at the solid/liquid interface: interaction forces and stability. Colloid Surf. A: Physicochem. Eng. Aspects A 139, 199-211.

Chen, C.Y., 1993. Comparative studies on the primary - treatment of municipal waste - water for ocean disposal. Water Sci. Technol. 28, 1-7.

Chhabra, R.P., Richardson, J.F., 1999. Non-Newtonian Flow in the Process Industries: Fundamentals and Engineering Applications. Butterworth-Heinemann, Oxford.

Gregory, J., 1985. The action of polymeric flocculants flocculation. In: Proceedings of Sedimentation and Consolidation, Engineering Foundation Conference, Georgia, USA, pp. 125-137.

Herschel, W.H., Bulkley, R., 1926. Konsistenzmessungen von Gummi-Benzol-Lösungen. Kolloid-Z 39, 291-300.
Huggins, M.L., 1942. The viscosity of dilute solutions of long-chain molecules IV Dependence on concentration. J. Am. Chem. Soc. 64, 2716-2718.

Jachimska, B., Jasinski, T., Warszynski, P., Adamczyk, Z., 2010. Conformations of poly(allylamine hydrochloride) in electrolyte solutions: experimental measurements and theoretical modelling. Colloid Surf. A: Physicochem. Eng. Aspects 355, 7-15.

Kemer, F.N., 1988. NALCO Water Handbook, 2nd ed. McGraw-Hill, New York.

Lauten, R.A., Nystrom, B., 1999. Linear and nonlinear viscoelastic properties of aqueous solutions of cationic polyacrylamides. Macrmol. Chem. Phys. 206 (6), 677-684.

Mortimer, D.A., 1991. Synthetic polyelectrolyte - a review. Polym. Int. 25, 29-41.

Ochoa, J.R., Sanz, F.J.E., Sasia, P.M., Garcia, A.S., de Apodaca, E.D., Rio, P., 2007. Synthesis of cationic flocculants by the inverse microemulsion copolymerization of acrylamide with $60 \%$ 2-acryloxyethyltrimethyl ammonium chloride in the monomer feed. II. Influence of the formulation composition, hydrophilic-lipophilic balance, starting polymerization temperature, and reaction time. J. Appl. Polym. Sci. 103, 186-197.

Rashidi, M., Blokhus, A.M., Skauge, A., 2010. Viscosity study of salt tolerant polymers. J. Appl. Polym. Sci. 117, 1551-1557.

Rasteiro, M.G., Garcia, F.A.P., Ferreira, P., Blanco, A., Negro, C., Antunes, E., 2008a. Evaluation of flocs resistance and reflocculation capacity using the LDS technique. Powder Technol. 183, 231-238.

Rasteiro, M.G., Garcia, F.A.P., Ferreira, P., Blanco, A., Negro, C., Antunes, E., 2008b. Effect of water cationic content on flocculation, flocs resistance and reflocculation capacity of PCC induced by polyelectrolytes. Ind. Eng. Chem. Res. 47, 6006-6013.

Schulz, G.V., Blaschke, F.J., 1941. Eine Gleichung zur Berechnung der Viscositätszahl für sehr kleine Konzentrationen, Molekulargewichtsbestimmungen an makromolekularen Stoffen, IX. J. Prakt. Chem. 158, 130-135.

Shubin, V., Linse, P., 1997. Self-consistent-field modeling of polyelectrolyte adsorption on charge-regulating surfaces. Macromolecules 30, 5944-5952.

Stoll, S., Chodanowski, P., 2002. Polyelectrolyte adsorption on an oppositely charged spherical particle. Chain rigidity effects. Macromolecules 35, 9556-9562.

Vanerek, A., Alince, B., Van de Ven, T.G.M., 2000. Colloidal behaviour of ground and precipitated calcium carbonate fillers: effects of cationic polyelectrolytes and water quality. J. Pulp Pap. Sci. 26, 135-139. 